Published by Al-Nahrain College of Medicine P-ISSN 1681-6579

E-ISSN 2224-4719

Email: iraqijms@colmed-alnahrain.edu.iq

http://www.colmed-alnahrain.edu.iq

http://www.iraqijms.net

Iraqi JMS 2018; Vol. 16(2)

\title{
The Effect of The Enzyme Replacement Therapy on The Kidney Function Tests and Serum Electrolyte Levels in Children with Gaucher Disease
}

\author{
Hiba A. Abdulhussein MSc, Firyal H. Al-Obaidi MSC, Hala S. Arif ${ }^{1} C A B P$
}

Dept. of Chemistry and Biochemistry, ${ }^{1}$ Dept. of Pediatrics, College of Medicine, Al-Nahrain University, Baghdad, Iraq

\begin{abstract}
Background Gaucher disease (GD) is an inherited autosomal recessive disease. It is most common in the Ashkenazi Jewish population. Many biomarkers might be involved in the etiology, pathogenesis, diagnosis and prognosis of GD in children. Most of them are related to complications due to an involvement of many organs such as liver, spleen and bones by this lysosomal storage disease that caused by a lack of the enzyme glucocerebrosidase.

Objective To investigate the role of kidney function test and electrolytes (urea, creatinine, sodium and potassium) level in the monitoring of the response for the treatment used for patients with GD in follow-up manner.

Methods A case control study was done on 67 children (32 males \& 35 females), age range from 2-14 years (mean \pm SD; $5.3 \pm 2.9$ ). The levels of sodium, potassium, urea and creatinine were measured in the samples of patients who were categorized as newly diagnosed untreated patients $(n=9)$, patients receiving enzyme replacement therapy (ERT) for 3-6 months ( $n=18), 6-12$ months $(n=20)$ and patients receiving ERT for more than one year $(n=20)$ and compared with twenty age-matched control subjects ( 9 males \& 11 females) age range from $2-14$ years (mean $\pm S D ; 5.55 \pm 3.05$ ).

Results The data indicated that the level of urea in GD patients $(23.39 \pm 4.71 \mathrm{mg} / \mathrm{dl})$ was significantly higher than that of age-matched controls $(17.5 \pm 3.05 \mathrm{mg} / \mathrm{dl})$. Non-significant differences were illustrated in the levels of sodium, potassium and creatinine. Negative significant $(p<0.05)$ correlations were obtained between the levels of urea $(r=-0.752 ; p<0.001)$ and creatinine $(r=-0.536 ; p<0.001)$ with the period of receiving ERT. Additionally, ANOVA test also revealed significant $(p<0.05)$ differences among the patients' subgroups in the levels of urea and creatinine. Results obtained from Receiver Operating Characteristic (ROC) curve revealed that urea and creatinine showed a high area under the curve (AUC), sensitivity and specificity $(0.939,77.8 \%$ and $85 \%$ for urea and $0.978,100 \%$ and $80 \%$ for creatinine respectively) in newly diagnosed GD patients in a comparison with control.

Conclusion The possibility of using urea and creatinine in the diagnosis and monitoring the effect of ERT on the GD patients.

Keywords Gaucher disease, macrophage, renal function test, urea, creatinine, sodium, potassium, enzyme replacement therapy, imiglucerase, $\beta$-glucocerebrosidase, glucocerebroside

Citation

Abdulhussein HA, Al-Obaidi FH, Arif HS. The effect of the enzyme replacement therapy on the kidney function tests and serum electrolyte levels in children with Gaucher disease. Iraqi JMS. 2018; 16(2): 182-190. doi: 10.22578/IJMS.16.2.10
\end{abstract}

List of abbreviations: ERT= enzyme replacement therapy, $\mathrm{GD}=$ Gaucher disease, $\mathrm{ROC}=$ Receiver Operating Characteristic, $\mathrm{AUC}=$ area under the curve, $\mathrm{TB}=$ tuberculosis, $\mathrm{ANOVA}=$ Analysis of variance.

\section{Introduction}

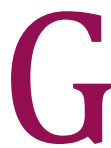

aucher Disease (GD) is considered as the most common autosomal recessive lysosomal storage disease that is caused by a deficiency in the enzyme $\beta$ - glucocerebrosidase leading to an accumulation in its substrate that called glucocerebroside, which is derived chiefly from membrane components of hematopoietic cells ${ }^{(1,2)}$. The accumulation of this substrate in lysosomes of macrophages cause a formation of pathological macrophages that called Gaucher cells, which 
considered as the main histological hallmark of the disease and are located in many organs ${ }^{(3,4)}$. The clinical manifestations and symptoms of this disease also ranged from asymptomatic individuals to massive hepatomegaly and splenomegaly in addition to hypersplenism and severe bone, and, occasionally, lung complications ${ }^{(5)}$. Neuronopathic involvements mainly occur in Gaucher disease types II and III leading to a wide variety of neurological manifestations in addition to visceral involvement ${ }^{(5,6)}$.

The treatment of choice for symptomatic Gaucher patients is enzyme replacement therapy (ERT) with imiglucerase (Cerezyme ${ }^{\circledR}$ ) (7). ERT showed to have a valuable effect on the GD symptoms and significantly cause a reduction in the organomegaly, in addition to an elevation in the levels of hemoglobin and platelet counts, and also improve children growth in with Gaucher patients ${ }^{(8)}$. On the other hand, the effect of ERT on bone or lung complications still less clear than other manifestations ${ }^{(9)}$.

This study tried to focus on the renal complications in GD patients since that there are a few case reports in the literature on the renal complications in association with GD. When renal abnormalities coexist with GD, a large variation in the clinical and laboratory manifestations were noticed including proteinuria, tubular defects or renal failure ${ }^{(3)}$. Additionally, autopsy of asymptomatic patients with GD showed Gaucher cells in the kidneys. It is still unclear whether the abnormalities in renal functions in these patients arise from their storage disease or whether during the course of a lifetime with GD, some patients seem to develop only one kind of kidney manifestation or another $(7,10)$.

To realize the effects of GD and ERT on the renal function, we undertook a case control study on patients with GD. The aim was to evaluate the effect of the disease and the treatment on some renal function parameters noninvasively.

\section{Methods}

A case control study was done on 67 children (32 males and 35 females), age range from 214 years (mean $\pm \mathrm{SD}$; $5.3 \pm 2.9$ ) who had GD recruited from Pediatric Department and Unit of Rare Diseases at Al-Imamein Al-Kadhimein Medical City, Gastroenterology and Hepatology Teaching Hospital, Children Welfare Hospital Consultation Clinic and Central Child's Teaching Hospital.

The levels of sodium, potassium, urea and creatinine were measured in the samples of 67 Gaucher patients who were categorized as newly diagnosed untreated patients $(n=9)$, patients receiving ERT for 3-6 months $(n=18)$, 6-12 months $(n=20)$ and patients receiving ERT for more than one year $(n=20)$ and compared with twenty age-matched control subjects (9 males and 11 females); age range from 2-14 years (mean \pm SD; $5.55 \pm 3.05$ ). A Control group with exclusion criteria that include patients with chronic infections, those suspected to have tuberculosis (TB) and patients with chronic inflammatory conditions as chronic arthritis in addition to other factors that affect enzyme activity.

The local Ethical Committee of the College of Medicine, University of Al-Nahrain, Baghdad, Iraq, approved this study. In addition, an informed written consent of participation in the study was signed by the parents or the legal guardians of the investigated subjects according to the Helsinki principles.

\section{Results}

The data indicated that the mean \pm standard deviation (SD) levels of urea in whole Gaucher patients $(23.39 \pm 4.71 \mathrm{mg} / \mathrm{dl})$ was significantly higher $(p<0.05)$ than that of age-matched controls $(17.5 \pm 3.05 \mathrm{mg} / \mathrm{dl})$. On the other hand, non-significant differences were illustrated in the levels of sodium, potassium and creatinine (table 1).

These parameters were remarkably associated with the period of receiving treatment with ERT that indicated by the decrease in the level of urea and creatinine with the increase in the duration of treatment as demonstrated by 
table (4) and also this finding confirmed by the negative significant $(p<0.05)$ correlations between the levels of urea $(r=-0.752 ; p<0.001)$ and creatinine $(r=-0.536 ; p<0.001)$ with the period of receiving treatment as demonstrated in table 2.
On the other hand a non-significant difference in the levels of sodium and potassium among all the studied groups were illustrated in table (3).

Table 1. The comparison of the studied parameters between Gaucher patients and control group by independent t-test

\begin{tabular}{cccc}
\hline & Control & Patients & \\
Parameter & $\mathbf{N}=\mathbf{2 0}$ & $\mathbf{N}=67$ & P value \\
& mean \pm SD & mean \pm SD & \\
\hline Sodium $(\mathbf{m E q} / \mathbf{l})$ & $137.95 \pm 1.23$ & $137.74 \pm 1.67$ & 0.615 \\
Potassium $(\mathbf{m E q} / \mathbf{l})$ & $4.13 \pm 0.2$ & $4.12 \pm 0.35$ & 0.982 \\
Urea $(\mathbf{m g} / \mathbf{d l})$ & $17.5 \pm 3.05$ & $23.39 \pm 4.71$ & $<0.0001$ \\
Creatinine $(\mathrm{mg} / \mathrm{dl})$ & $0.27 \pm 0.09$ & $0.3 \pm 0.08$ & 0.163 \\
\hline
\end{tabular}

Table 2. Correlation between the studied parameters in whole Gaucher patients

\begin{tabular}{cccccc}
\hline & & Potassium & Urea & Creatinine & Duration of treatment \\
\hline \multirow{2}{*}{ Sodium } & $\mathrm{r}$ & 0.057 & -0.128 & -0.339 & 0.189 \\
& $\mathrm{p}$ & 0.647 & 0.303 & 0.005 & 0.125 \\
\hline \multirow{2}{*}{ Potassium } & $\mathrm{r}$ & & 0.007 & -0.045 & -0.091 \\
& $\mathrm{p}$ & & 0.957 & 0.716 & 0.464 \\
\hline \multirow{2}{*}{ Urea } & $\mathrm{r}$ & & & 0.376 & -0.734 \\
& $\mathrm{p}$ & & & 0.007 & $<0.001$ \\
\hline \multirow{2}{*}{ Creatinine } & $\mathrm{r}$ & & & & -0.605 \\
& $\mathrm{p}$ & & & & $<0.001$ \\
\hline
\end{tabular}

r: Pearson correlation coefficient, P: Significance

Additionally, ANOVA test also revealed the effect of ERT that is indicated by the significant $(p<0.05)$ differences among the patients' subgroups in the levels of urea and creatinine (table 5). Results obtained from Receiver Operating Characteristic (ROC) curve revealed that urea and creatinine showed a high area under the curve (AUC), sensitivity and specificity $(0.939,77.8 \%$ and $85 \%$ for urea and $0.978,100 \%$ and $80 \%$ for creatinine respectively) in newly diagnosed GD patients in a comparison with control and variable values of area under the curve (AUC), sensitivity and specificity in whole GD and other GD patient subgroups as demonstrated in tables (6-10). 
Table 3. Sodium and Potassium levels in control, patients with 3-5 months, 6-12

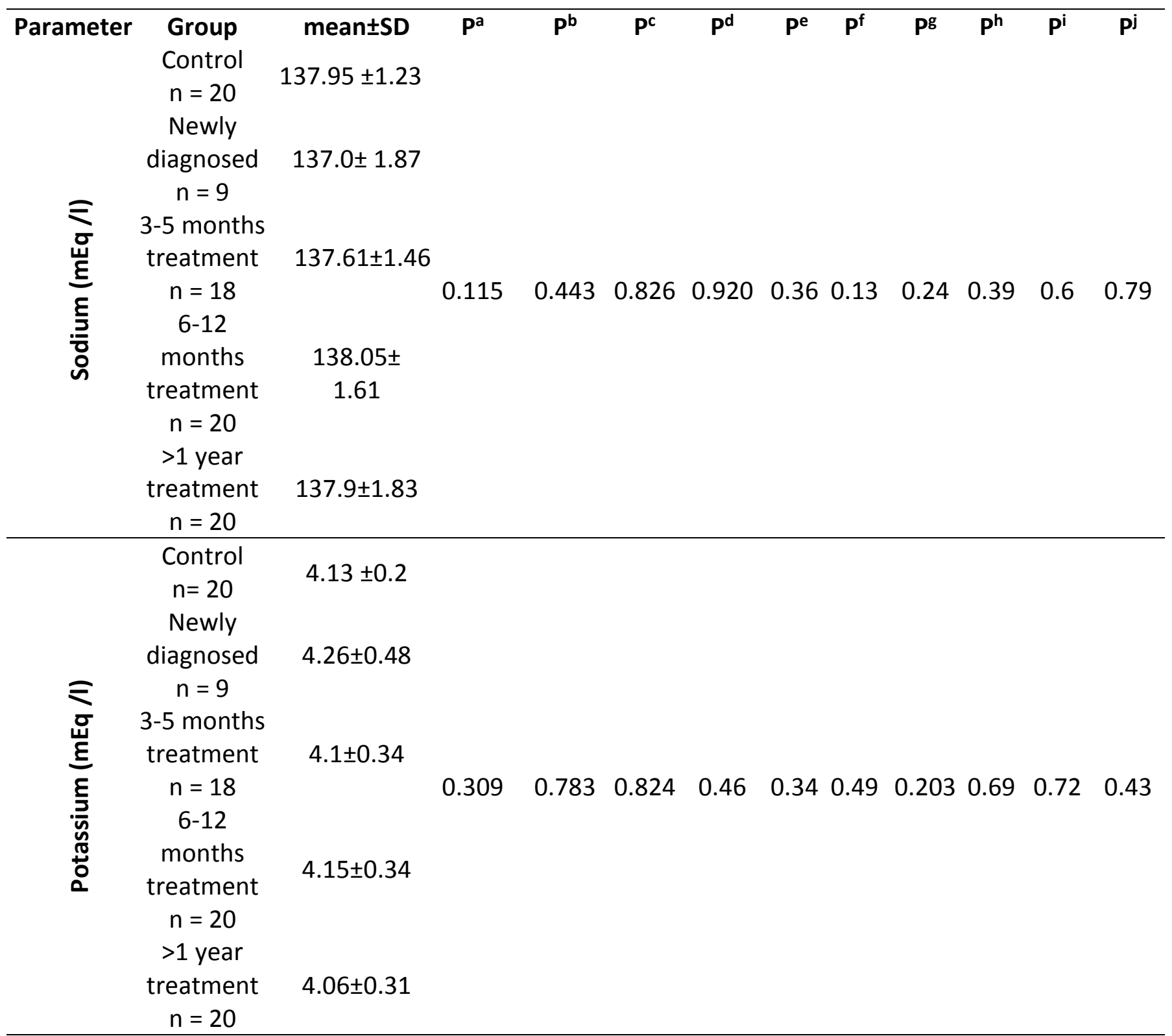

Pa value between newly diagnosed patients and control

$\mathrm{Pb}$ value between patients with 3-5 months treatment and control

Pc value between patients with 6-12 months treatment patients and control

Pd value between patients with $>1$ year treatment and control.

Pe value between newly diagnosed patients and patients with 3-5 months treatment

Pf value between newly diagnosed patients and patients with 6-12 months treatment

$\mathrm{Pg}$ value between newly diagnosed patients and patients with $>1$ year treatment

$\mathrm{Ph}$ value between patients with 3-5 months and patients with 6-12 months treatment

Pi value between patients with 3-5 months and patients with $>1$ year treatment

Pj value between patients with 6-12 months and patients with $>1$ year treatment 
Table 4. Urea and Creatinine levels in control, patients with 3-5 months, 6-12 months and $>1$ year treatment groups

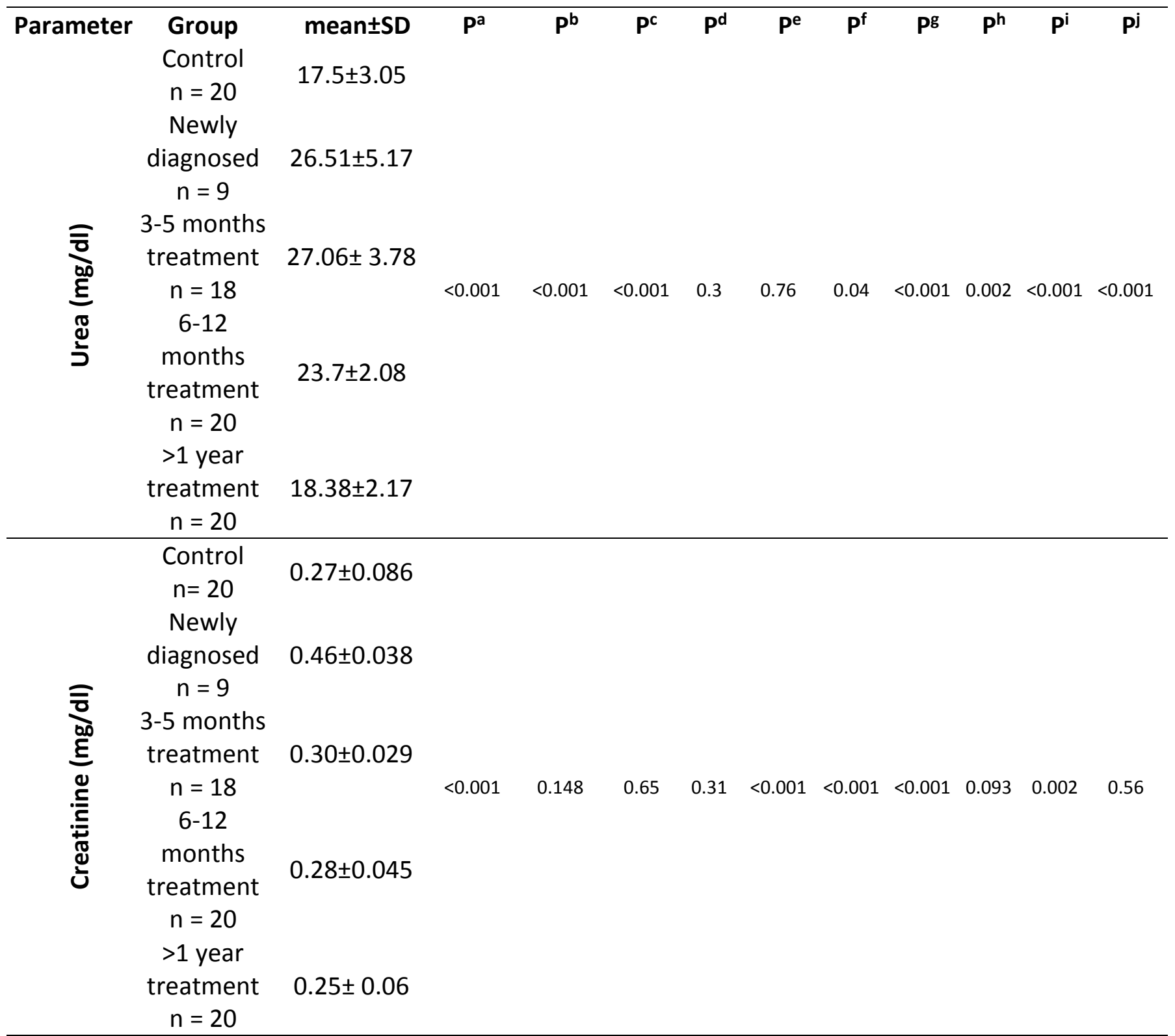

Pa value between newly diagnosed patients and control

$\mathrm{Pb}$ value between patients with 3-5 months treatment and control

Pc value between patients with 6-12 months treatment patients and control

$P d$ value between patients with $>1$ year treatment and control.

Pe value between newly diagnosed patients and patients with 3-5 months treatment

Pf value between newly diagnosed patients and patients with 6-12 months treatment

Pg value between newly diagnosed patients and patients with $>1$ year treatment

Ph value between patients with 3-5 months and patients with 6-12 months treatment

$\mathrm{Pi}$ value between patients with 3-5 months and patients with $>1$ year treatment

Pj value between patients with 6-12 months and patients with $>1$ year treatment 
Table 5. The comparison of the studied parameters between Gaucher patients' subgroups by ANOVA test

\begin{tabular}{|c|c|c|c|c|c|c|}
\hline Parameter & $\begin{array}{c}\text { Control } \\
n=20 \\
\text { mean } \pm S D\end{array}$ & $\begin{array}{c}\text { Newly } \\
\text { diagnosed } \\
n=9 \\
\text { mean } \pm \text { SD }\end{array}$ & $\begin{array}{c}\text { 3-5 months } \\
\text { treatment } \\
n=18 \\
\text { mean } \pm S D\end{array}$ & $\begin{array}{c}\text { 6-12 months } \\
\text { treatment } \\
n=20 \\
\text { mean } \pm S D\end{array}$ & $\begin{array}{c}>1 \text { year } \\
\text { treatment } \\
n=20 \\
\text { mean } \pm S D\end{array}$ & $P$ value \\
\hline $\begin{array}{l}\text { Sodium } \\
\text { (mEq/I) }\end{array}$ & $137.95 \pm 1.23$ & $137 \pm 1.87$ & $137.61 \pm 1.46$ & $138.05 \pm 1.61$ & $137.9 \pm 1.83$ & 0.442 \\
\hline $\begin{array}{c}\text { Potassium } \\
\text { (mEq/l) }\end{array}$ & $4.13 \pm 0.2$ & $4.26 \pm 0.48$ & $4.1 \pm 0.34$ & $4.15 \pm 0.34$ & $4.06 \pm 0.31$ & 0.575 \\
\hline $\begin{array}{l}\text { Urea } \\
\text { (mg/dl) }\end{array}$ & $17.5 \pm 3.05$ & $26.51 \pm 5.17$ & $27.06 \pm 3.78$ & $23.7 \pm 2.08$ & $18.38 \pm 2.17$ & $<0.001$ \\
\hline $\begin{array}{l}\text { Creatinine } \\
\text { (mg/dl) }\end{array}$ & $0.27 \pm 0.09$ & $0.46 \pm 0.04$ & $0.30 \pm 0.03$ & $0.28 \pm 0.05$ & $0.25 \pm 0.06$ & $<0.001$ \\
\hline
\end{tabular}

Table 6. ROC curve results for all studied parameters in patients comparing with control

\begin{tabular}{ccccc}
\hline Parameters & AUC & Sensitivity (\%) & Specificity (\%) & Cut-off value \\
\hline Sodium (mEq/l) & 0.563 & 70.0 & 48.0 & 137.5 \\
Potassium (mEq/l) & 0.53 & 55.0 & 58.0 & 4.15 \\
Urea (mg/dl) & 0.865 & 82.0 & 80.0 & 18.5 \\
Creatinine (mg/dl) & 0.615 & 76.0 & 60.0 & 0.235 \\
\hline
\end{tabular}

Table 7. ROC curve results for all studied parameters in newly diagnosed patients comparing with control

\begin{tabular}{ccccc}
\hline Parameters & AUC & Sensitivity (\%) & Specificity (\%) & Cut-off value \\
\hline Sodium (mEq/l) & 0.717 & 70.0 & 78.0 & 137.5 \\
Potassium (mEq/l) & 0.6 & 55.6 & 90.0 & 4.35 \\
Urea (mg/dl) & 0.939 & 77.8 & 85.0 & 22.15 \\
Creatinine (mg/dl) & 0.978 & 100 & 80.0 & 0.35 \\
\hline
\end{tabular}

Table 8. ROC curve results for all studied parameters in newly diagnosed patients comparing with patients with 3-6 months treatment

\begin{tabular}{ccccc}
\hline Parameters & AUC & Sensitivity (\%) & Specificity (\%) & Cut-off value \\
\hline Sodium (mEq/l) & 0.664 & 83.0 & 56.0 & 136.5 \\
Potassium (mEq/l) & 0.611 & 55.6 & 83.3 & 4.35 \\
Urea (mg/dl) & 0.506 & 88.9 & 33.3 & 22.65 \\
Creatinine (mg/dl) & 1 & 100 & 100 & 0.365 \\
\hline
\end{tabular}


Table 9. ROC curve results for all studied parameters in patients with 3-6 months treatment comparing with patients with 6-12 months treatment

\begin{tabular}{ccccc}
\hline Parameters & AUC & Sensitivity (\%) & Specificity (\%) & Cut-off value \\
\hline Sodium (mEq/l) & 0.586 & 65.0 & 61.1 & 137.5 \\
Potassium (mEq/l) & 0.551 & 35.0 & 83.3 & 4.35 \\
Urea (mg/dl) & 0.797 & 61.1 & 95.0 & 25.5 \\
Creatinine (mg/dl) & 0.657 & 83.3 & 50.0 & 0.275 \\
\hline
\end{tabular}

Table 10. ROC curve results for all studied parameters in patients with 6-12 months treatment comparing with patients with $>1$ year treatment

\begin{tabular}{ccccc}
\hline Parameters & AUC & Sensitivity (\%) & Specificity (\%) & Cut-off value \\
\hline Sodium (mEq/l) & 0.519 & 65.0 & 35.0 & 137.5 \\
Potassium (mEq/l) & 0.568 & 45.0 & 70.0 & 4.15 \\
Urea (mg/dl) & 0.945 & 90.0 & 85.0 & 21.15 \\
Creatinine (mg/dl) & 0.703 & 100 & 65.0 & 0.205 \\
\hline
\end{tabular}

\section{Discussion}

Generally, most of the previous studies stated that the Gaucher patients didn't have a renal involvement and the level of serum urea and creatinine in addition to electrolytes such as sodium and potassium were normal ${ }^{(3,11)}$ with some exceptions $(7,12)$ that occurs rarely. This clinical manifestation of organ dysfunction directly related to massive Gaucher cells infiltration ${ }^{(7,10)}$ such as appeared in the present study in which a significant increase in the serum urea were observed. On the other hand, non-significant differences in the levels of creatinine, sodium and potassium were reported in Gaucher patients in comparison with control subjects as demonstrated in table (1).

\section{Sodium and potassium}

According to results obtained in table (3), it was demonstrated that there was a nonsignificant difference in the levels of sodium and potassium among all the studied groups that may indicate a non-significant effect of the GD and also the treatment with ERT on the level of these electrolytes in agreement with previous studies ${ }^{(3,11)}$. The present study also revealed that the level of sodium and potassium didn't affect by treatment as revealed by a non-significant result obtained by ANOVA test in table ${ }^{(5)}$ and also confirmed by the non-significant correlation with the period of treatment as shown in table (2). Also, ROC curve results illustrated that these two electrolytes cannot be considered as biomarkers for GD due to low AUC, sensitivity and specificity in all patients in comparison with controls.

\section{Urea}

In contrast to tests for the assessment of sodium, potassium and also creatinine, urea showed a different pattern in the present study, which also differ from the most previous studies $(3,11)$ in that urea levels showed a significant higher level compared to controls as demonstrated in table (1). Additionally table (4) also revealed that the levels of urea in newly diagnosed untreated patients, patients treated with ERT for 3-6 months and patients treated for 6-12 months were significantly higher than that of control while the level of urea return to a normal level, which is comparable to that of control after receiving the treatment for more than year and also noticed that there was a non-significant 
differences between newly diagnosed untreated patient and patients received treatment for 3-6 months.

These results may indicate that the treatment with ERT for 6-12 months may cause a significant reduction in the renal complication and cause a urea level restoration to the normal levels after more than one year of treatment. This suggestion also confirmed by the significant difference among all patients' subgroups as demonstrated by ANOVA test in table (5) and also confirmed by the significant negative correlation between urea level and the period of treatment that demonstrated in table (2) and ROC curve results obtained in tables (6-10), which indicate that urea level can be considered as an excellent biomarker for GD patients either treated or untreated with ERT.

As mentioned previously, the assumed explanation of these abnormalities is the infiltration of glomeruli by Gaucher cells ${ }^{(7)}$.

\section{Creatinine}

In contrast to urea, the levels of creatinine in all studied groups were normal and showed a non-significant difference between Gaucher patients and control and the only interesting finding was the significant difference between the newly diagnosed untreated patients and controls after that the level of creatinine return to a level comparable to that of control in patients receiving treatment which may indicate that the level of creatinine is more sensitive to treatments and respond more quickly than urea which is also revealed by a significant differences between untreated and treated patients as shown in table (4).

ANOVA test results in table (5) also prove the above finding of significant differences between all patient subgroups. In addition to that, the significant negative correlation between the creatinine levels and the period of treatment that illustrated in table (2) also ensure the effect of ERT on the renal complication together with ROC curve results which revealed that creatinine can be considered as an excellent biomarker for GD especially for newly diagnosed untreated patients and also to monitor the treatment in the first 3-6 months of treatment (tables 6-10).
Finally, according to the previous data and data provided in this study, it was concluded that kidney involvement in Gaucher patients may be monitored by urea and creatinine levels which provide a complementary picture about the all steps of kidney restoration to normal function given that creatinine is more sensitive for treatment in the first 6 months of treatment while the significant differences in urea level become more obvious after the 6 months of treatment.

In conclusion, kidney involvement in Gaucher patients can be monitored by urea and creatinine levels which provide a complementary picture about the all steps of kidney restoration to normal function given that creatinine is more sensitive for treatment in the first 6 months of treatment while the significant differences in urea level become more obvious after the 6 months of treatment. Sodium and potassium levels showed to be non-significantly affected by the disease and treatment with ERT.

\section{Acknowledgments}

Authors are grateful to staff of the Department of Chemistry and Biochemistry, College of Medicine, Al-Nahrain University for their facilities in performing this study, Department and Unit of Rare Diseases at Al Imamein AlKadhimein Medical City, Gastroenterology and Hepatology Teaching Hospital, Children Welfare Hospital Consultation Clinic, Central Child's Teaching Hospital and College of Education, Ministry of Science and Technology.

\section{Authors contribution}

Abdulhussein: Performing laboratory measurements. Al-Obaidi: Writing of the manuscript and statistical analysis. $\mathrm{Dr}$ Arif: Providing patient samples and diagnosing autistic patient cases.

\section{Conflict of interest}

Authors have no conflict of interests.

\section{Funding}

The work was not supported or funded by any drug company. 


\section{References}

1. Alcalay RN, Dinur $T$, Quinn $T$, et al. Comparison of Parkinson risk in Ashkenazi Jewish patients with Gaucher disease and GBA heterozygotes. JAMA Neurol. 2014; 71(6): 752-7. doi: 10.1001/jamaneurol.2014.313.

2. Kanneganti M, Kamba A, Mizoguchi E. Role of chitotriosidase (chitinase 1 ) under normal and disease conditions. J Epithel Biol Pharmacol. 2012; 5: 1-9.

3. Becker-Cohen R, Elstein D, Abrahamov A, et al. A comprehensive assessment of renal function in patients with Gaucher disease. Am J Kidney Dis. 2005; 46(5): 837-44. doi: 10.1053/j.ajkd.2005.07.042.

4. Nagral A. Gaucher Disease. J Clin Exp Hepatol. 2014; 4(1): 37-50. doi: 10.1016/j.jceh.2014.02.005.

5. Weiss K, Gonzalez A, Lopez G, et al. The clinical management of Type 2 Gaucher disease. Mol Genet Metab. 2015; 114(2): 110-22. doi: 10.1016/j.ymgme.2014.11.008.

6. Linari S, Castaman G. Clinical manifestations and management of Gaucher disease. Clin Cases Miner Bone Metab. 2015; 12(2): 157-64. doi: 10.11138/ccmbm/2015.12.2.157.

7. Stirnemann J, Belmatoug N, Camou F, et al. A review of Gaucher Disease pathophysiology, clinical presentation and treatments. Int J Mol Sci. 2017; 18(2). pii: E441. doi: 10.3390/ijms18020441.
8. Andrade-Campos $M$, Alfonso $P$, Irun $P$, et al. Diagnosis features of pediatric Gaucher disease patients in the era of enzymatic therapy, a nationalbase study from the Spanish Registry of Gaucher Disease. Orphanet J Rare Dis. 2017; 12(1): 84. doi: 10.1186/s13023-017-0627-z.

9. Chen M, Wang J. Gaucher disease: review of the literature. Arch Pathol Lab Med. 2008; 132(5): 851-3. doi: $10.1043 / 1543-$

2165(2008)132[851:GDROTL]2.0.CO;2.

10. Kim MJ, Suh JT, Lee HJ, et al. Simultaneous detection of Gaucher's disease and renal involvement of nonHodgkin's lymphoma: the first Asian case report and a review of literature. Ann Clin Lab Sci. 2012; 42(3): 293-301.

11. Modak D, Roy $S$, Nath $U$, et al. Type 1 and type 3 Gaucher disease in two siblings in a family: 2 unusual case reports. J Clin Diagn Res. 2015; 9(2): OD01-2. doi: 10.7860/JCDR/2015/8493.5507.

12. Siegal A, Gutman A, Shapiro MS, et al. Renal involvement in Gaucher's disease. Postgrad Med J. 1981; 57(668): 398-401.

\section{Correspondence to Hiba A. Abdulhussein \\ E-mail: hibaabdulhussein@yahoo.com}

Received Aug. 15 2017

Accepted Nov. 16 2017 